Part of the Journal of Research of the National Bureau of Standards

\title{
Preparation of Mannuronic Lactone From Algin
}

\author{
By Harriet L. Frush and Horace S. Isbell
}

\begin{abstract}
A simple procedure is reported for the preparation of crystalline mannuronic lactone from algin. The algin is converted to alginic acid, which is hydrolyzed by the action of concentrated sulfuric acid followed by dilute acid. In contrast to prior methods, the lactone is crystallized directly from the hydrolyzate of alginic acid without the intermediate separation of the amorphous barium salt or the cinchonine salt. The yield is from 25 to 30 percent of the alginic acid used. The preparation and properties of crystalline sodium mannuronate and potassium mannuronate monohydrate are also described.
\end{abstract}

\section{Introduction}

Mannuronic acid, one of the three naturally occurring uronic acids, is of particular interest because it plays an important role in the structure of many marine plants, and is the chief constituent of algin, a product of growing commercial importance. It has been prepared by others in the form of the crystalline lactone $[1,2]^{1}$ and two modifications of the free acid [3]. It may be separated most readily as the lactone, but prior methods of preparation are so unsatisfactory that it has been isolated only in small quantities. To make the substance more available for study and research, a simple procedure has been developed for the preparation of mannuronic lactone directly from the hydrolyzates of alginic acid. Inasmuch as the raw materials used are commercially available at relatively low cost, the preparation of mannuronic lactone in any desired quantity now becomes practicable.

Crystalline mannuronic lactone was first prepared by Nelson and Cretcher [1] by the acid hydrolysis of alginic acid, followed by the intermediate preparation of amorphous barium mannuronate and crystalline cinchonine mannuronate.

The method is laborious and the yield is very low. Schoeffel and Link [2] separated mannuronic lactone from the hydrolyzate of alginic acid by means of a carefully purified amorphous barium salt without recourse to the cinchonine

\footnotetext{
${ }_{1}$ Figures in brackets indicate the literature references at the end of this
} paper. salt. Fractional precipitation of the unhydrolyzed polymannuronates and various clarifications, filtrations, and concentrations were required to give the crystalline lactone in yields calculated to be 8.3 percent of the theoretical (based on the uronic acid content of the alginic acid). In both methods the hydrolyzates were neutralized by prolonged heating with barium carbonate. ${ }^{2}$ This treatment is necessary for complete neutralization, but it causes considerable decomposition of the mannuronic acid.

In the foregoing procedures it was necessary to prepare and purify one or even two intermediate compounds because it was impossible to crystallize mannuronic lactone directly from the crude

2 Nelson and Cretcher neutralized the hot hydrolyzate with barium carbonate, but later changed the procedure to include partial neutralization with barium hydroxide at room temperature followed by treatment with barium carbonate for 2 hours at boiling temperature.

\section{Contents}

I. Introduction

II. Experimental details_............... 322

1. Preparation of alginic acid_......... 322

2. Preparation of mannuronic lactone from alginic acid_... 322

III. New crystalline salts of mannuronic acid_._._. 323

1. Sodium mannuronate___.__ _ _ 323

2. Potassium mannuronate monohydrate -324

IV. References . . . . 
hydrolyzate. Apparently the difficulty in crystallizing mannuronic lactone arises, first, from incomplete hydrolysis of the polysaccharide, and second, from decomposition reactions incident to the hightemperature neutralization of the hydrolyzate with barium carbonate. The hydrolysis is complicated by the fact that alginic acid is only slowly cleaved by hot acids, whereas under the conditions of the reaction, the product, mannuronic acid, is partially decomposed with the formation of carbon dioxide and furfural. Nelson and Cretcher found that on boiling for 18 hours with distilled water, alginic acid loses 10 percent of the theoretical amount of carbon dioxide; on boiling for 5 hours with 12-percent hydrochloric acid it loses 86 percent of the theoretical amount. To avoid the undesirable decomposition reaction, they recommended hydrolysis with 80-percent sulfuric acid at room temperature for 5 days. Schoeffel and Link, however, hydrolyzed by boiling with 2.5percent sulfuric acid for 15 hours.

Thus, it seemed probable that if a more complete hydrolysis of algin were effected, and the hot neutralization of the hydrolyzate were avoided, it might be possible to crystallize mannuronic lactone directly from the hydrolyzate. With this object in view, various methods were tried for hydrolyzing alginic acid and for neutralizing the hydrolyzate. The experiments led to a simple procedure for the hydrolysis, followed by removal of the sulfuric acid at room temperature, without conversion of the mannuronic acid to a salt. After concentration of the hydrolyzate, crystalline mannuronic lactone separates in yields of 25 to 30 percent of the uronic acid content of the alginic acid.

In the course of the investigation, the possibility of separating mannuronic acid as a crystalline salt was considered, and attempts were made to find a suitable substance. Crystalline sodium mannuronate and potassium mannuronate were prepared, and their properties are reported here. The salts crystallize readily but are too soluble for use in the preparation of mannuronic lactone:

\section{Experimental Details}

\section{Preparation of Alginic Acid}

Alginic acid is an intermediate in the industrial preparation of algin, but at present it is not commercially available. It may be readily prepared from commercial algin by the following procedure: $1 \mathrm{~kg}$ of algin is stirred for 2 hours with a solution consisting of 3 liters of alcohol, ${ }^{3} 2$ liters of water, and $350 \mathrm{ml}$ of concentrated hydrochloric acid. The crude alginic acid is then separated by vacuum filtration in a large Büchner funnel, or more conveniently in an 8-liter conical percolator equipped with a perforated plate and a cheesecloth filter pad. It is then stirred with a solution containing 1 liter of alcohol, 3 liters of water, and 100 $\mathrm{ml}$ of concentrated hydrochloric acid. After 2 hours the alginic acid is again separated by vacuum filtration, and during a period of several days is washed by gravity in the funnel or percolator with a large volume of water. When the wash water is free from chlorides, the material is washed with alcohol and then dried in air and finally in a vacuum at $60^{\circ} \mathrm{C}$. The yield of alginic acid is approximately $700 \mathrm{~g}$. When a product prepared by the above method was boiled for 8 hours with

\footnotetext{
${ }^{3}$ Methyl, ethyl, or isopropyl alcohol may be used.
}

18-percent hydrochloric acid, it gave carbon dioxide equivalent to 90 percent of mannuronic anhydride.

\section{Preparation of Mannuronic Lactone From Alginic Acid}

Eighty grams of alginic acid is added quickly to $100 \mathrm{ml}$ of ice-cold, concentrated sulfuric acid. ${ }^{4}$ The pasty mass is kept in an ice bath and is stirred constantly with a heavy glass rod for 10 minutes. It is stored in a refrigerator for 18 hours and then is thoroughly mixed with 8 liters of water. The diluted mixture is boiled under reflux for 16 hours, ${ }^{5}$ cooled to room temperature, and treated with a quantity of calcium carbonate equivalent to the sulfuric acid. The $\mathrm{pH}$ of the resulting solution should be 2.3 . If not, the acidity is adjusted by the addition of either calcium carbonate or dilute sulfuric acid.

\footnotetext{
- When alginic acid is mixed with concentrated sulfuric acid, the product turns straw-colored, then brown, and finally black. The dark mixture, when diluted with acetic anhydride, appears deep blue; with acetic acid, reddishviolet; and with water or alcohol, yellowish-brown. Apparently the dark color originally formed is not caused by carbonization, but arises from a highly-colored substance.

i A Glas-col mantle provides a convenient source of heat without danger of overheating the material. Boiling chips should be used to prevent bumping.
} 

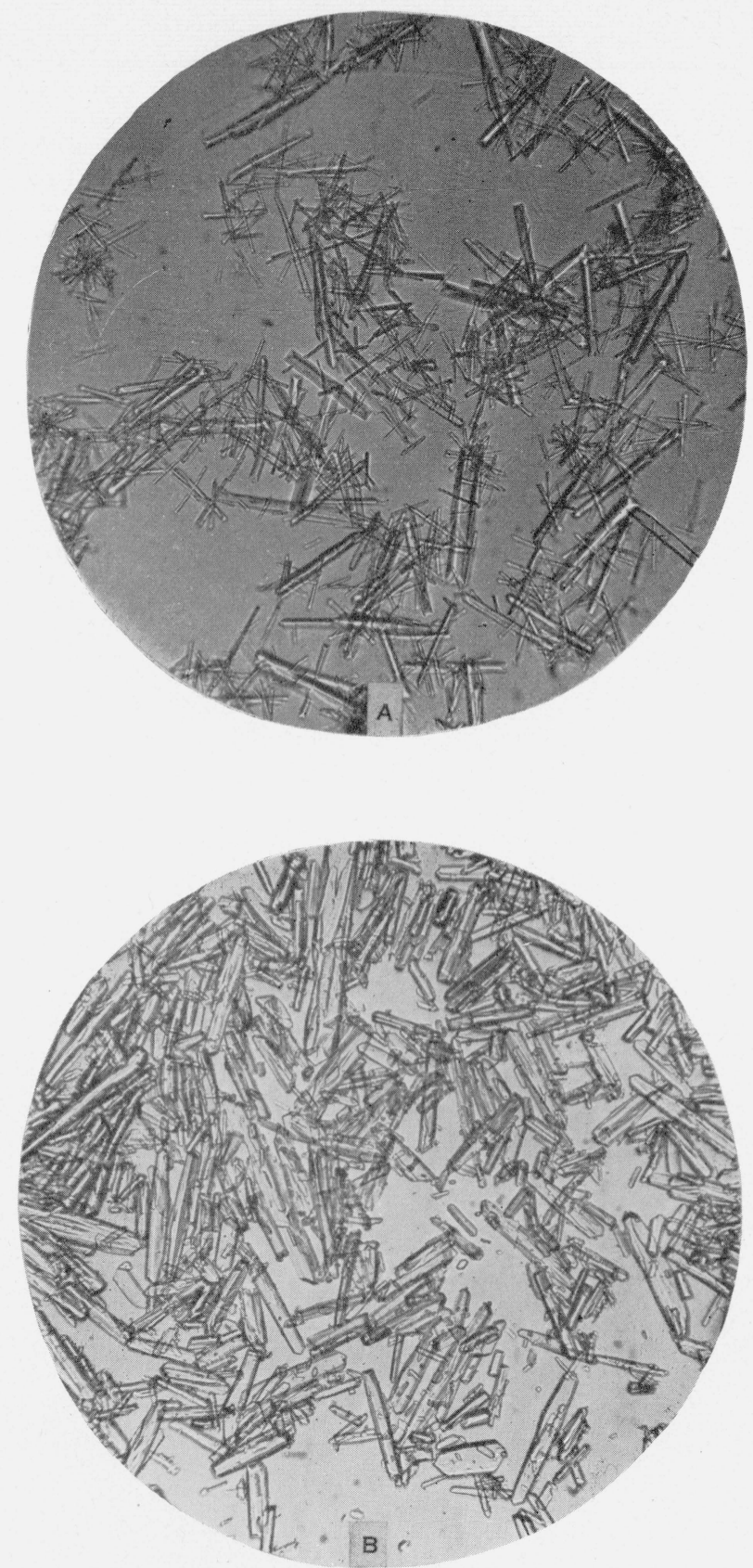

Figure 1.-A, Sodium mannuronate; $B$, potassium mannuronate monohydrate 
The mixture is filtered with the aid of decolorizing carbon, and the residue on the filter is washed and discarded. The filtrate is evaporated at $40^{\circ} \mathrm{C}$ or less under reduced pressure to a volume of about $400 \mathrm{ml}$. The calcium sulfate that crystallizes during the evaporation is separated, washed with hot water until free from reducing substances, and discarded. The solution and washings are combined and evaporated under reduced pressure to a volume of about $100 \mathrm{ml}$. At this point the calcium sulfate is again separated, washed, and discarded, and the solution is evaporated under reduced pressure to a thick sirup (about $40 \mathrm{ml}$ ). Ordinarily, mannuronic lactone begins to crystallize during the final evaporation. The sirup is diluted with approximately 2 volumes of acetic acid, seeded (if crystallization has not already started), and allowed to stand in the refrigerator for 18 hours. ${ }^{6}$ The crystals are then separated by filtration and washed, first with a mixture consisting of 3 parts of acetic acid and 1 part of water, and finally with acetone. ${ }^{7}$ The product contains a small quantity of calcium sulfate, which is removed by dissolving the lactone in hot water and filtering the solution with the aid of a decolorizing carbon. The lactone crystallizes almost quantitatively when the solution is evaporated in vacuum or in a stream of dry air.

In numerous preparations by the above method in which the alginic acid used had a mannuronic anhydride content of 90 percent, the main crop of crystals weighed between 20 and $24 \mathrm{~g}$ and contained less than $1 \mathrm{~g}$ of calcium sulfate. In a typical preparation, the crude lactone from $80 \mathrm{~g}$ of alginic acid weighed $21.2 \mathrm{~g}$, contained $0.36 \mathrm{~g}$ of calcium sulfate, and had a specific rotation of $+87.2^{\circ}$ (30 minutes after dissolution in water). After recrystallization from water, the product weighed 20.1 g. $[\alpha]_{\mathrm{D}}^{20}=+92.0^{\circ}$ (30 minutes), whereas for pure mannuronic lactone, $[\alpha]_{\mathrm{D}}^{20}=$ $92.2^{\circ}$ (30 minutes) [4]. The yield of the recrystallized lactone was 25.1 percent of the weight of the alginic acid, or 27.9 percent of the theoretical.

- Nearly as satisfactory results can be obtained by the addition of a suitable quantity of either acetone or dioxane. As crude sirups of mannuronic lactone gradually darken and decompose, they should be kept at a low temperature.

7 A small, rather crude second crop may sometimes be obtained by concentration of the mother liquor and subsequent dilution with acetic acid.

\section{New Crystalline Salts of Mannuronic Acid}

\section{Sodium Mannuronate}

Twenty grams of mannuronic lactone was dissolved in water at room temperature and slightly less than the theoretical amount of sodium bicarbonate $(10.5 \mathrm{~g})$ was added in portions with stirring. The solution was filtered with the aid of a decolorizing carbon, and concentrated under reduced pressure to a volume of about $35 \mathrm{ml}$. Sufficient ethyl alcohol was added to cause a faint turbidity that was discharged by the addition of a drop of water. The solution was seeded and allowed to crystallize while in motion. Alcohol was added from time to time as crystallization proceeded until the volume of the mixture was $100 \mathrm{ml}$. After 2 days the crystals of sodium mannuronate were collected on a filter, washed with aqueous ethyl alcohol, and finally with 95-percent alcohol. They were dried in a vacuum at room temperature over calcium chloride. The yield of crude, yellow-colored sodium mannuronate was 17.0 g. After two successive recrystallizations by dissolving the crystals in water at room temperature and treating the solution in the manner described above, the compound was obtained as a white crystalline powder composed of slender prisms (fig. 1). Further recrystallization produced no change in optical rotation.

Analysis: Calculated for $\mathrm{C}_{6} \mathrm{H}_{9} \mathrm{O}_{7} \mathrm{Na}$ : C, 33.33; $\mathrm{H}, 4.20$. Found: $\mathrm{C}, 33.4 ; \mathrm{H}, 4.3$.

When dissolved in water containing carbon dioxide, the compound exhibits a small mutarotation (table 1 ), which at $20^{\circ} \mathrm{C}$ is substantially complete in 2 minutes. $[\alpha]_{\mathrm{D}}^{20}=-6.6^{\circ}$ (water, $\mathrm{c}=4$ ) (30 minutes).

TABLE 1.-Mutarotation of sodium mannuronate in water saturated with carbon dioxide

\begin{tabular}{|c|c|c|}
\hline \multicolumn{3}{|c|}{$2 \mathrm{~g}$ per $100 \mathrm{ml}$ of solution at $20^{\circ} \mathrm{C}$ read in a } \\
\hline Time & Observed reading & $[\alpha]]_{D}^{20}$ \\
\hline Minutes & $\circ S$ & \\
\hline 0.5 & -1.65 & -14.3 \\
\hline 7 & -1.35 & -11.7 \\
\hline 9 & -1.17 & -10.1 \\
\hline 1.0 & -0.97 & -8.4 \\
\hline $1.5 \ldots$ & -.80 & -6.9 \\
\hline $1.8 \ldots$ & -.79 & -6.8 \\
\hline $2.1 \ldots \ldots \ldots$ & -.76 & -6.6 \\
\hline $2.4 \ldots$ & -.77 & -6.7 \\
\hline 2.8 & -.75 & -6.5 \\
\hline 3.1 & -.82 & -7.1 \\
\hline 5.0 & -.76 (constant) & -6.6 \\
\hline
\end{tabular}




\section{Potassium Mannuronate Monohydrate}

Twenty grams of mannuronic lactone were dissolved at room temperature in water saturated with carbon dioxide, and the solution was treated with $11.2 \mathrm{~g}$ of potassium bicarbonate. Potassium mannuronate monohydrate was then crystallized in the manner dsecribed for sodium mannuronate. The crude material ( $16 \mathrm{~g}$ ) was recrystallized twice from water and alcohol. The optical rotation of the substance was not altered by further recrystallization from water alone.

Analysis: Calculated for $\mathrm{C}_{6} \mathrm{H}_{2} \mathrm{O}_{7} \mathrm{KH}_{2} \mathrm{O}: \mathrm{C}, 28.80$; $\mathrm{H}, 4.43$. Found: C, 29.1; H, 4.6.

The crude potassium mannuronate had a yellow color, but the pure compound was obtained in long, colorless plates or prisms (fig. 1). Like sodium mannuronate, it exhibits a rapid mutaro- tation, which is complete in less than 3 minutes (table 2). $[\alpha]_{\mathrm{D}}^{20}=-5.6^{\circ}$ (water, $c=4$ ) (30 minutes).

TABLE 2.-Mutarotation of potassium mannuronate monohydrate in water saturated with carbon dioxide

\begin{tabular}{|c|c|c|}
\hline \multicolumn{3}{|c|}{$2 \mathrm{~g}$ per $100 \mathrm{ml}$ of solution at $20^{\circ} \mathrm{C}$ read in a } \\
\hline Time & Observed reading & {$[\alpha]_{\mathrm{D}}^{20}$} \\
\hline Minutes & ${ }^{\circ} S$ & \\
\hline $0.5 \ldots \ldots$ & -2.12 & -18.3 \\
\hline .7 & -1.83 & -15.8 \\
\hline $.9 \ldots$ & -1.68 & -14.5 \\
\hline $1.0 \ldots \ldots$ & -1.40 & -12.1 \\
\hline $1.3 \ldots \ldots$ & -1.07 & -9.3 \\
\hline $1.8 \ldots$ & -0.91 & -7.9 \\
\hline $2.0 \ldots \ldots$ & -.82 & -7.1 \\
\hline $2.4 \ldots \ldots$ & -.75 & -6.5 \\
\hline $2.9 \ldots \ldots$ & -.69 & -6.0 \\
\hline $5.0 \ldots \ldots \ldots$ & -.65 (constant) & -5.6 \\
\hline
\end{tabular}

\section{References}

[1] W. L. Nelson and L. H. Cretcher, J. Am. Chem. Soc. 54, 3409 (1932).

[2] E. Schoeffel and K. P. Link, J. Biol. Chem. 95, 213 (1932).

[3] E. Schoeffel and K. P. Link, J. Biol. Chem. 100, 397 (1933).
[4] H. S. Isbell and H. L. Frush, J. Research NBS 37, 43 (1946) RP1727.

Washington, September 3, 1946. 